\title{
Design, simulation and construction of a delay wire chamber
}

Cite as: AIP Conference Proceedings 1935, 070003 (2018); https://doi.org/10.1063/1.5025984

Published Online: 27 February 2018

Aytül Adıgüzel, Ezgi Ergenlik, Saime Gürbüz, Zehra İstemihan, V. Erkcan Özcan, and Gökhan Ünel

ARTICLES YOU MAY BE INTERESTED IN

KAHVE Laboratory RF circulator and transmission line project

AIP Conference Proceedings 1935, 070002 (2018); https://doi.org/10.1063/1.5025983

Design, simulation and construction of a Wire Chamber electronics

AIP Conference Proceedings 1815, 130005 (2017); https://doi.org/10.1063/1.4976484

Integrability of the one dimensional Schrödinger equation

Journal of Mathematical Physics 59, 022105 (2018); https://doi.org/10.1063/1.5023242

\section{Conference Proceedings}

Get $30 \%$ off all print proceedings! 


\title{
Design, Simulation And Construction Of A Delay Wire Chamber
}

\author{
Aytül Adıgüzel $^{1}$, Ezgi Ergenlikk ${ }^{2, a)}$, Saime Gürbüz ${ }^{2}$, Zehra İstemihan, ${ }^{2, *}$ \\ V. Erkcan Özcan ${ }^{2}$ and Gökhan Ünel ${ }^{3}$ \\ ${ }^{1}$ Istanbul University, Physics Dept., 34119 Istanbul, Turkey \\ ${ }^{2}$ Boğaziçi University, Dept. of Physics, 34342 İstanbul, Turkey \\ ${ }^{3}$ University of California, Irvine, Physics and Astronomy Dept., 92697 California, USA \\ a)Corresponding author: ezgiergenlik@gmail.com
}

\begin{abstract}
Delay wire chamber (DWC) is a relatively inexpensive gaseous detector that enables tracking of charged particles with high resolution. Here, design and simulation of a DWC that is suitable for beam diagnostics measurements at low-energy accelerators are described. We report on the mechanical construction of such a chamber at the Kandilli Detector, Accelerator and Instrumentation Laboratory, and the tests performed with cosmic muons and radioactive sources.
\end{abstract}

\section{INTRODUCTION}

Gaseous particle detectors, from Geiger-Müller tubes to the wire chambers, have a long history of scientific applications [1]. Their working principle relies on the ionization of gases by the incoming charged particles, amplification of the generated primary charges with the help of an applied high voltage, and the collection of electrons or their images on thin wires. The type of the detector in which the signal is collected from multiple anode wires is called the multi wire proportional chamber (MWPC). Delay wire chambers, developed by the Beam Instrumentation Group at CERN for measuring the beam profiles at linear accelerators, work with similar principles and can be considered as simplified versions of MWPCs [2]. Their first difference is on the cathodes: in a DWC a set of closely spaced wires, placed perpendicularly to the anode wires, act as the cathode instead of a conductor plane. When the drifted electrons reach the anode wires they create image charges on the cathode wires. The second difference of the DWCs is in their read-out: in MWPC all the signals are read from each individual wire separately, while the DWC signals are accumulated on a delay line which is then read from both ends (Figure a). The timing difference between the two signals $(\Delta t)$ can be converted into position information by a simple linear relation: $x=\alpha \times \Delta t+\beta$ where the calibration coefficients $\alpha$ and $\beta$ are to be determined experimentally. The typical position precision of a DWC is about 200 microns [3].

With multiple new accelerator projects (for example [4]) being carried out in Turkey, a need has arisen for various beam diagnostics equipment, including DWCs. In order to address this need, a chamber was designed, simulated and constructed at the Kandilli Detector, Accelerator and Instrumentation (KAHVE) Laboratory at Boğaziçi University. In order to also allow for the future possibility of replacing aging wire chambers used at CERN, the initial design was selected to be similar to those built there [5].

\section{TECHNICAL DETAILS AND DESIGN}

The cathode layer consists of grounded $100 \mu \mathrm{m}$-thick $\mathrm{Cu}$-Be wires that are positioned $2 \mathrm{~mm}$ of each other. $2-3 \mathrm{kV}$ voltage is applied on the anode wires, which are positioned $4 \mathrm{~mm}$ apart from each other and oriented perpendicular to the cathode wires. There are 3 layers (cathode-anode-cathode configuration) for each dimension in which tracking is to be performed and the whole system consists of two such configurations that are placed at 90 degrees for 2D

${ }^{*}$ Currently at Koç University, Dept. of Physics, 34450 Sarıer, İstanbul, Turkey.

Turkish Physical Society 33rd International Physics Congress (TPS33) AIP Conf. Proc. 1935, 070003-1-070003-5; https://doi.org/10.1063/1.5025984 Published by AIP Publishing. 978-0-7354-1627-7/\$30.00 

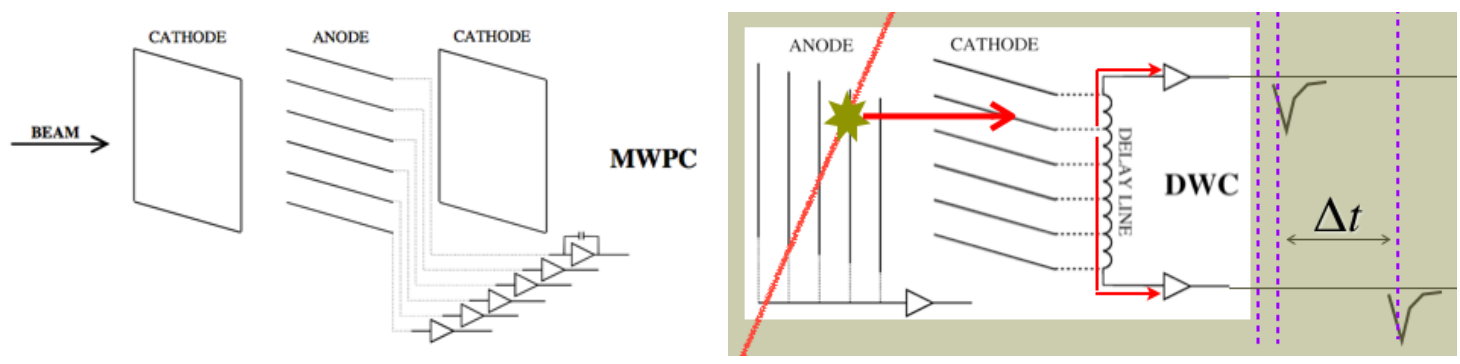

FIGURE 1: Left: MWPC read-out schematic [5]. Right: DWC delayed read-out schematic.

position measurement. The signal is collected from the combination of pairs of cathode wires that lie on top of each other on the two layers, increasing the amplitude of the signal. Collected signal from the anode wires are used only for obtaining a trigger signal, although a scintillator-PMT setup can also be used for trigger. Initially a 50\%-50\% gas mix of $\mathrm{CO}_{2}$-Ar was used, but the concentration of $\mathrm{Ar}$ was later increased to $70 \%$ to increase the signal amplitude.

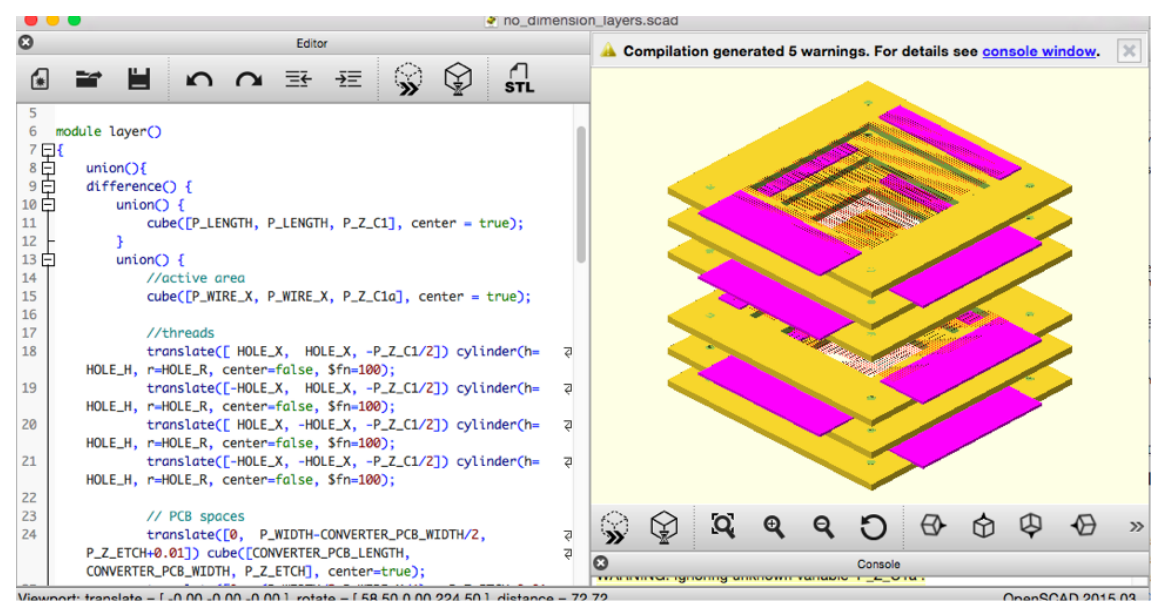

FIGURE 2: OpenSCAD DWC design example: the script can be seen on the left and the generated 3D graphics can be seen on the right.

The mechanical design of the layers has been done with the open source program OpenSCAD [6]. This program allows the user to do 3D modeling of the DWC layers with a basic script file, allowing full control over the shape and the design parameters. Another script has been written for imploring the needs of an enduser and automatically producing the OpenSCAD input file that contains the design parameters, enabling endusers to easily design DWCs based on their particular needs.

\section{SIMULATIONS}

The simulations have been performed with the open source software Garfield++ [7]. It allows users to simulate both the electric field (for simple configurations) and the interactions of the particles in gaseous tracking detectors. A current limitation of this program is that it is not possible to add perpendicular cathode wires, so very thin cathode strips have been placed between two anode wires for the electric field simulation (Figure c). The simulated signal generated by an electron of $1 \mathrm{GeV}$ momentum, and sent directly towards the chamber starting $5 \mathrm{~mm}$ away from it, can be seen in Figure d. 


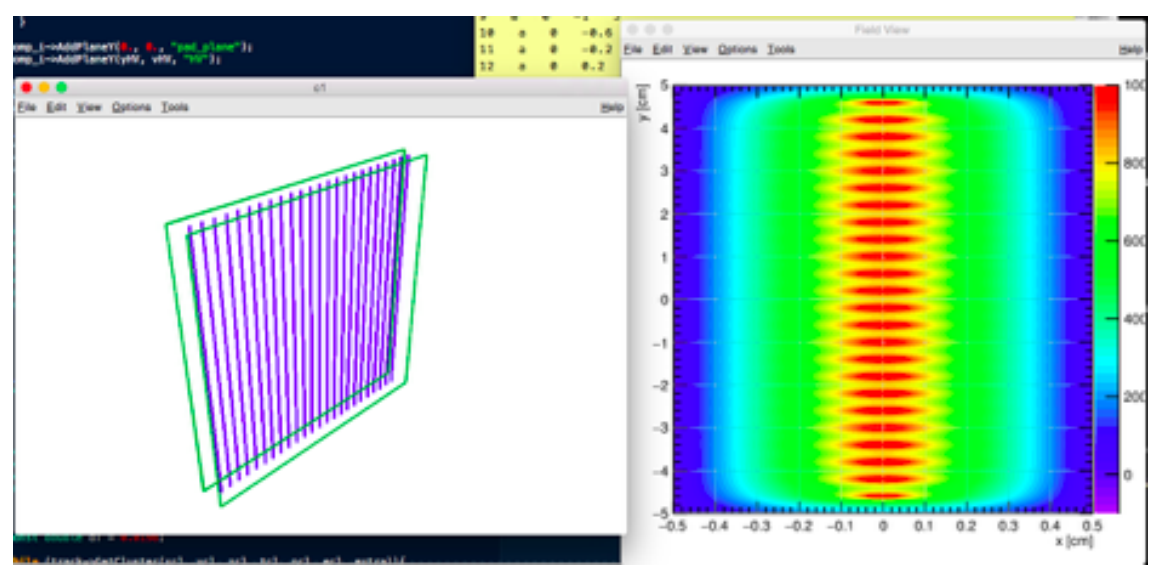

FIGURE 3: Left: Sketch of the chamber. Right: Simulated electric field near the anode wires, which have been placed perpendicular to the plane of the paper.
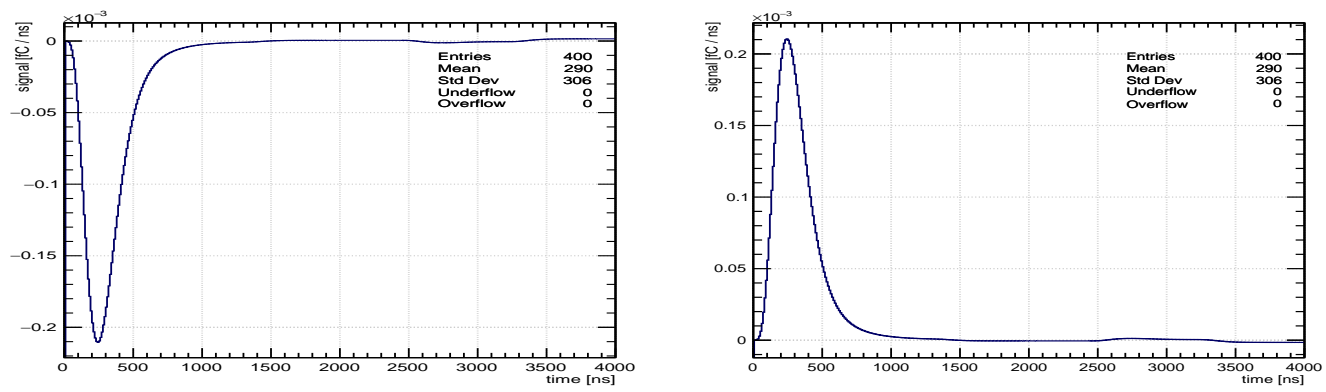

FIGURE 4: The simulated current as a function of time generated by the passing of an electron: in the anode (left) and the cathode (right) wires.

\section{CONSTRUCTION}

FR4 (flame retardant grade 4) was chosen because of the mechanical stress caused by the wires and the high voltage in the anode wires for drifting electrons. FR4 was partitioned into $19 \times 19 \mathrm{~cm}$ squares using a rotary saw and a milling cutter. In addition to cutting out at the center of each square $10 \times 10 \mathrm{~cm}$ windows at the center of each square, over which the wires would be placed, depressions on the sides were prepared as slots where read-out connector PCBs could be glued with epoxy. $20 \mu \mathrm{m} \mathrm{Au}$ plated $W$ and $100 \mu \mathrm{m} \mathrm{Cu}$-Be wires were selected as anode and cathode, respectively. For stretching the anode and cathode wires we used $20 \mathrm{~g}$ and $105 \mathrm{~g}$ sinkers (Figure e). After the wires were soldered, two steps were taken to clean the chamber frame and the wires: First, all layers were baked at $150^{\circ} \mathrm{C}$ in a dry heat oven for 2 hours and secondly these were cleaned in an ultrasonic alcohol bath for 30 minutes.

\section{TEST RESULTS}

Initially the chamber was tested with a simple non-amplifier circuit consisting of a capacitor and resistance. The first result can be seen in Figure f. After it was determined that chamber was working, an amplifier circuit was implemented similar to the ones that are used at CERN [8]. When the initial trials were not found to be completely satisfactory in terms of stability and noise, an improved circuit was designed and built. This new design was tested up to $10 \mathrm{MHz}$ on a breadboard and no considerable noise or reflections were observed. 

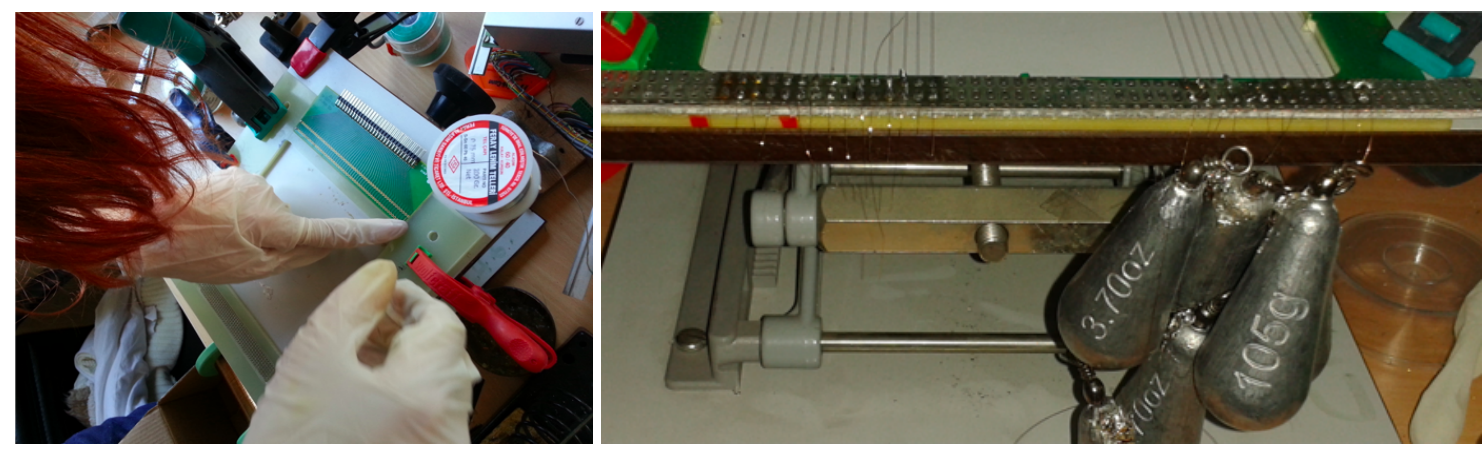

FIGURE 5: Left: Stretching and welding the wires. Right: the weights used for providing uniform tension to the wires.

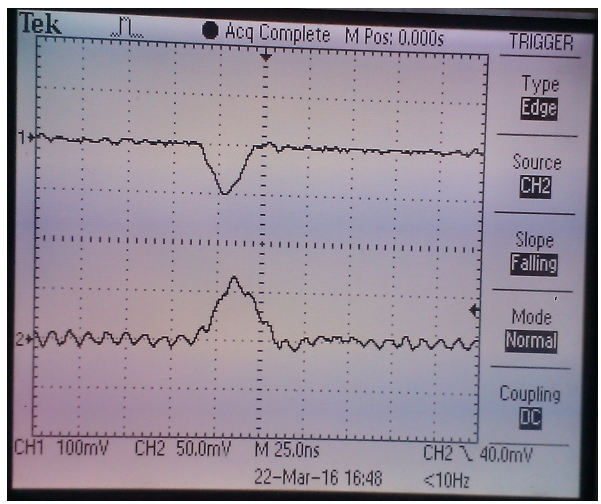

FIGURE 6: First signal from the anode and cathode wires generated by cosmic particles.

The constructed chamber was also tested with Co-60 radioactive sources at CERN's gaseous detectors laboratory. Larger signal pulses were observed as compared to the performances of the DWCs in use at CERN (Figure g). A completed DWC with the new amplifier circuit is in preparation for beam tests at national accelerator facilities.

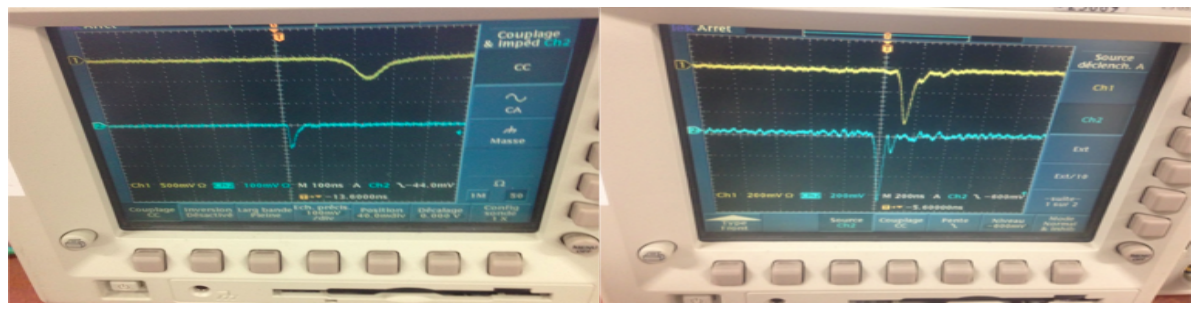

FIGURE 7: Example signals observed during the tests at CERN. The delays between the two ends of the delay wire are visible.

\section{ACKNOWLEDGMENTS}

The authors would like to thank J. Spanggaard for useful suggestions and his assistance at CERN's gaseous detectors laboratory, and to Ali Bozbey, Taha Yasin Posos and Alperen Yüncü for their contributions to the experimental setup and to the production of the amplifier circuit. This work has been supported by TÜBİTAK under the grant number 114F467. A. Adıgüzel acknowledges past financial support from Boğaziçi University BAP project $16 \mathrm{~B} 03$ P6. 


\section{References}

[1] G. Charpak and F. Sauli, Multiwire Proportional Chambers and Drift Chambers, NIM 1621979.

[2] A. Manarin and G. Vismara, The Delay Wire Chamber Description, LEP BI-TA Note 85-3.

[3] S. Gurbuz et al., A wire chamber for educational purposes, arXiv:1409.0523 [physics.ins-det], Sep 2014.

[4] G. Turemen et al., SPP Beamline Design and Beam Dynamics, Proceedings of the 5th International Particle Accelerator Conference (IPAC'14), 15-20 June 2014, THPME050, p. 3338, arXiv:1406.3066 [physics.accph], Apr 2015.

[5] J. Spanggaard, Delay Wire Chambers, A Users Guide, SL-Note-98-023, CERN - SL DIVISION.

[6] OpenSCAD The Programmers Solid 3D CAD Modeller, http://www . openscad.org (accessed November 17, 2017).

[7] Garfield, a drift-chamber simulation program, CERN Program Library entry W5050, http://garfield. web. cern. ch (accessed November 17, 2017).

[8] Z. İstemihan on behalf of the Boun DWC team, Design, simulation and construction of a Wire Chamber electronics, AIP Conference Proceedings 1815, 130005 (2017). 